\title{
Andrographolide inhibits growth of acute promyelocytic leukaemia cells by inducing retinoic acid receptor-independent cell differentiation and apoptosis
}

\begin{abstract}
OBJECTIVES: The growth inhibiting potential of andrographolide was evaluated in three acute promyelocytic leukaemia cell line models (HL-60, NB4 and all-trans retinoic acid (ATRA)-resistant NB4-R2). METHODS: In elucidating the mechanisms of growth inhibition, a special emphasis was placed on assessing the induction of differentiation and apoptosis by andrographolide in the primary acute promyelocytic leukaemia NB4 cells. KEY FINDINGS: The compound was 2- and 3-fold more active in inhibiting the growth of HL-60 and NB4-R2 cells compared with NB4 cells, respectively. At IC50 (concentration at which growth of 50\% of the cells (compared with medium only treated control cells) is inhibited; 4.5 microM) the compound exhibited strong cell-differentiating activity in NB4 cells, similar to ATRA (IC50 1.5 microM). In the presence of a pure retinoic acid receptor antagonist AGN193109, the growth inhibition of NB4 cells by ATRA was reversed, whereas the activity of andrographolide was not affected. This clearly suggested that andrographolide's cell differentiating activity to induce growth inhibition of NB4 cells most likely occurred via a retinoic acid receptor-independent pathway. At higher concentration (2xIC50), andrographolide was an efficient inducer of apoptosis in NB4 cells. CONCLUSIONS: Taken together, these results suggest andrographolide and its derivatives, apparently with a novel cell differentiating mechanism and with ability to induce apoptosis, might be beneficial in the treatment of primary and ATRA-resistant acute promyelocytic leukaemia.
\end{abstract}

Keyword: Andrographolide; Apoptosis; Cell differentiation; Promyelocyte; Retinoic acid receptor alpha 